\title{
Considerations to prevent the breakdown and loss of fruit carotenoids during extraction and analysis in Musa
}

\author{
Mark W. Davey*, Ifigeneia Mellidou, Wannes Keulemans \\ Laboratory for Fruit Breeding and Biotechnology, Division of Crop Biotechnics, Katholieke Universiteit Leuven, De Croylaan 42, Heverlee, Leuven, Belgium
}

\section{A R T I C L E I N F O}

\section{Article history:}

Received 19 February 2009

Received in revised form 4 June 2009

Accepted 5 June 2009

Available online 10 June 2009

\section{Keywords:}

Provitamin A carotenoids

Banana

Oxidation

Isomerisation

HPLC

Stability

\begin{abstract}
A B S T R A C T
The impact of treatments aimed at improving the robustness of protocols for the analysis of carotenoids in fruit of banana and plantain were examined. Neither the inclusion of polyvinylpolypyrrolidine in the extraction buffer, nor vigorous homogenisation with glass beads influenced recoveries or chromatographic profiles. By contrast, heating lead to losses of up to $53 \%$ and to the formation of degradation products that are no longer detectable on our RP-HPLC system. Carotenoid extracts are unstable and most sensitive to exposure to light. However, even in the dark at $-20^{\circ} \mathrm{C}$ and in the presence of antioxidants breakdown rates of around 5\% per day were observed.
\end{abstract}

(C) 2009 Elsevier B.V. All rights reserved.

\section{Introduction}

Plants are the major dietary source of carotenoids for humans and have many health-related properties. However, the provitamin A carotenoids (pVACs) which are broken down in the body to yield retinol, the active form of vitamin A [1,2], are of particular interest due to the widespread prevalence of vitamin A deficiencies worldwide [3]. Understandably therefore there is much interest in developing crops biofortified for pVACs contents to help combat these problems [4,5]. Quantitatively, the major pVACs are $\alpha$ - and $\beta$-carotene, $\beta$-cryptoxanthin and cis-isomers of $\alpha$ - and $\beta$-carotene. Recent studies from our lab indicate that there is a huge range of diversity in Musa fruit carotenoids and pVACs contents [6,7] and that existing pVACs-rich Musa varieties could have significant longterm beneficial health impacts for vitamin A deficient populations, particularly in regions where Musa spp. are a staple part of the diet $[4,6,8]$. Within the framework of a widescale screening program we have already established protocols for the sampling, extraction and direct HPLC analysis of Musa fruit carotenoids [7,9]. However, plant carotenoids are particularly sensitive to degradation during extraction and this can lead to cis-trans isomerisation, oxidative cleavage and/or epoxidation of the carotenoid backbone [10-13]. While the deleterious effects of heat, acids, light, etc., on carotenoids have

\footnotetext{
* Corresponding author. Tel.: +32 16 322414; fax: +32 16323966.

E-mail address: Mark.Davey@biw.kuleuven.be (M.W. Davey).
}

regularly been cited, the extent of the impact of these factors has rarely been determined. Here we quantify the effect of several postharvest procedures reported to influence carotenoid stability and recoveries, including the effects of heat and light during sample preparation and report on updates and modifications to protocols for carotenoid analysis in Musa fruit to improve robustness and reliability.

\section{Experimental}

\subsection{Musa fruit samples}

Experiments were carried out using the varieties "Batard", "Mbouroukou-1", "Oonoonoo kengoa" and "Henderneyargh". Fruits were harvested immature at ripening stage 1 [14], and then peeled, sliced, frozen and lyophilised before shipping to the laboratory in Leuven. Prior to analysis, the lyophilised samples were crushed to a fine powder in a pestle and mortar in liquid nitrogen under reduced light and stored in sealed $50 \mathrm{~mL}$ Falcon tubes at $-20^{\circ} \mathrm{C}$ in the dark [9].

\subsection{Materials}

HPLC-grade tetrahydrofuran (THF) and tert-butyl methyl ether (t-BME) were obtained from Sigma-Aldrich (Bornem, Belgium), HPLC-grade methanol $(\mathrm{MeOH})$ from LabScan (Dublin, Ireland). Lutein, all-trans $\beta$-carotene (t-BC), trans $\beta$-8-apocarotenal (apocarotenal), butylated hydroxytoluene (BHT), triethylamine and 
insoluble polyvinylpolypyrrolidine (PVPP) were all obtained from Sigma-Aldrich.

\subsection{Extraction}

50-100 mg aliquots of powdered Musa fruit pulp were homogenised for $30 \mathrm{~s}$ at maximum speed with 3-5 glass beads in a 'FastPrep' reciprocal shaker (Savant Instruments, New York, USA) in 5-10 volumes/g dry weight of ice-cooled extraction buffer consisting of THF:MeOH, 1:1 (v/v), 0.25\% BHT and 2\% insoluble PVPP [6,9]. Following centrifugation $\left(14,000 \times g\right.$ for $20 \mathrm{~min}$ at $\left.4{ }^{\circ} \mathrm{C}\right)$, the pellet was re-extracted twice more with 5-10 volumes of THF:MeOH, 1:1 (v/v), 0.25\% BHT without PVPP.

\subsection{RP-HPLC analysis}

The combined supernatants were directly analysed by RP-HPLC using a Waters Alliance, 2690 Separations System fitted with an autosampler at $8^{\circ} \mathrm{C}$, a pulse dampener and a $996 \mathrm{UV}$-vis photodiode array detector (Waters, MA, USA). The entire system was controlled and data collected and integrated using the Millenium 4.0 software package. Extracts were resolved on a $150 \times 4.6 \mathrm{~mm}$, YMC $\mathrm{C}_{30}$ 3- $\mu \mathrm{m}$ particle size HPLC column (Achrom, Zulte, Belgium), using an optimised 24 min linear gradient of $2-50 \% \mathrm{t}-\mathrm{BME}$ in $\mathrm{MeOH}$ at $1.0 \mathrm{~mL} / \mathrm{min}$ and regenerated with 95\% $\mathrm{t}-\mathrm{BME}$ in $\mathrm{MeOH}$. All buffers contained $0.05 \%$ BHT and $0.05 \%$ triethylamine. Peaks were quantified at $450 \mathrm{~nm}$ using a freshly prepared standard curve of t-BC in extraction buffer as previously described [9] and identified on the basis of their characteristic absorption spectra and retention times relative to known standards where available [9,15-18]. Apocarotenal at a final concentration of $0.002 \mu \mathrm{g} / \mathrm{mL}$ was added as an internal standard to correct for differences in extraction volumes.

\subsection{Impact of PVPP during extraction}

Triplicate aliquots of lyophilised fruit pulp from the plantain Mbouroukou-1 were homogenised in cooled extraction buffer either with or without $2 \%$ insoluble PVPP. In both cases two additional extractions of the pellet were carried out in extraction buffer without PVPP as described above. The combined extracts were directly analysed by RP-HPLC.

\subsection{Impact of heat during extraction}

Replicate aliquots of a lyophilised sample of Batard fruit pulp were extracted with ice-cold extraction buffer containing PVPP followed by a single heating step in a heating block at $85^{\circ} \mathrm{C}$ for between 10 and $90 \mathrm{~min}$ in a closed screw-capped polypropylene container. After cooling, samples were centrifuged at $14,000 \times \mathrm{g}$ for $20 \mathrm{~min}$ at $4 \mathrm{C}$ and the pellets re-extracted twice more with ice-cold extraction buffer before RP-HPLC analysis.

\subsection{Impact of homogenisation on recoveries and oxidation}

Replicate aliquots of powdered, lyophilised fruit pulp from the cultivars Oonoonoo kengoa and Henderneyargh were subjected either to vigorous shaking with glass beads in cold extraction buffer using a 'FastPrep' reciprocal shaker (30 s at maximum speed) during each of the 3 rounds of solvent extraction, for 2 rounds, during only the first extraction or not shaken at all.

\subsection{Stability of carotenoid solutions and extracts}

Freshly prepared $1 \mathrm{~mL}$ aliquots of $0.002 \mu \mathrm{g} / \mathrm{mL}$ solutions of t-BC and apocarotenal standards in THF:MeOH, 1:1 (v/v), 0.1\% BHT were stored for up to 10 days in either clear glass sample vials at room temperature in direct daylight, in amber glass vials in the dark at room temperature or in amber vials in the dark at $4{ }^{\circ} \mathrm{C}$. Samples were periodically analysed by $\mathrm{C}_{30}$ RP-HPLC to monitor the impact of storage conditions on sample stability.

$1 \mathrm{mg} / \mathrm{mL}$ stock solutions of t-BC and apocarotenal standards in THF:MeOH, 1:1 (v/v), 0.1\% BHT, were prepared and aliquots of these solutions stored at $-20^{\circ} \mathrm{C}$ in the dark. These aliquots were used daily to prepare standard curves over the range $0.002-0.1 \mathrm{mg} / \mathrm{mL}$ and the response curves compared over time.

\section{Results and discussion}

The literature contains frequent references to procedures that impact on carotenoid stability and extraction efficiencies [19-23]. However, the extent of these effects has generally not been quantified, and there are no reports for similar studies in Musa.

\subsection{Tissue sample storage}

It has been reported that lyophilisation of tissues leads to carotenoid degradation, due to the increased porosity of samples and an increased exposure to air [23]. We previously demonstrated that there are no significant differences in the recoveries of total pVACs from either lyophilised or fresh Musa fruit pulp tissues [9]. There was further no significant loss or breakdown of carotenoids during storage of lyophilised tissue in the dark at $-20^{\circ} \mathrm{C}$, whereas with frozen pulp tissues variable losses and a gradual browning (oxidation) indicative of residual peroxidase and polyphenoloxidase activities were observed. We therefore recommend storing tissues at $-20^{\circ} \mathrm{C}$ in lyophilised form in sealed containers in the dark.

\subsection{Impact of PVPP during extraction}

Musa tissues are known to be particularly rich in phenolic compounds which can potentially interfere with carotenoid extraction and/or analysis [24]. The inclusion of $2 \%$ insoluble PVPP in the extraction buffer to complex phenolics had no significant influence on absolute recoveries $(66.0 \pm 3.5$ and $63.8 \pm 5.0 \mathrm{nmol}$ total pVACs $/ \mathrm{g}$ dry weight for samples of Mbouroukou- 1 prepared with and without PVPP, respectively). There were further no differences in the RP-HPLC profiles demonstrating that PVPP has no significant effect on carotenoid analysis in Musa. However, PVPP did help to prevent occasional fouling of the HPLC columns and was therefore included as standard in the extraction buffer for the first extraction.

\subsection{Impact of heat during extraction}

The literature contains reports indicating that carotenoid contents are higher from tissue that has been boiled or heated $[19,25]$ suggesting that a heating step during extraction could improve carotenoid yields. We previously reported that a short heat treatment had no significant influence on recoveries of pVACs from Musa when using ethanol as an extraction solvent, although there was a slight increase in the proportion of 13 -cis- $\beta$-carotene [9]. Here, however, results clearly show that a longer period of heating significantly reduces the recoveries of all carotenoid compounds present, despite the inclusion of $0.25 \%$ BHT in the extraction buffer (Fig. 1).

At no point are extraction efficiencies improved and after a single 90 min heating step at $85^{\circ} \mathrm{C}$ only $52-53 \%$ of the initial t-AC and $\mathrm{t}-\mathrm{BC}$ concentrations remained. Heating has also been reported to selectively promote the formation of 13 -cis isomers [10,19], but we observed only a minor increase in the levels of 13-cis isomers of $\alpha$ - (c-AC) and $\beta$-carotene (c-BC) and only after extended heating. Importantly, despite large decreases in pVACs concentrations, heat- 


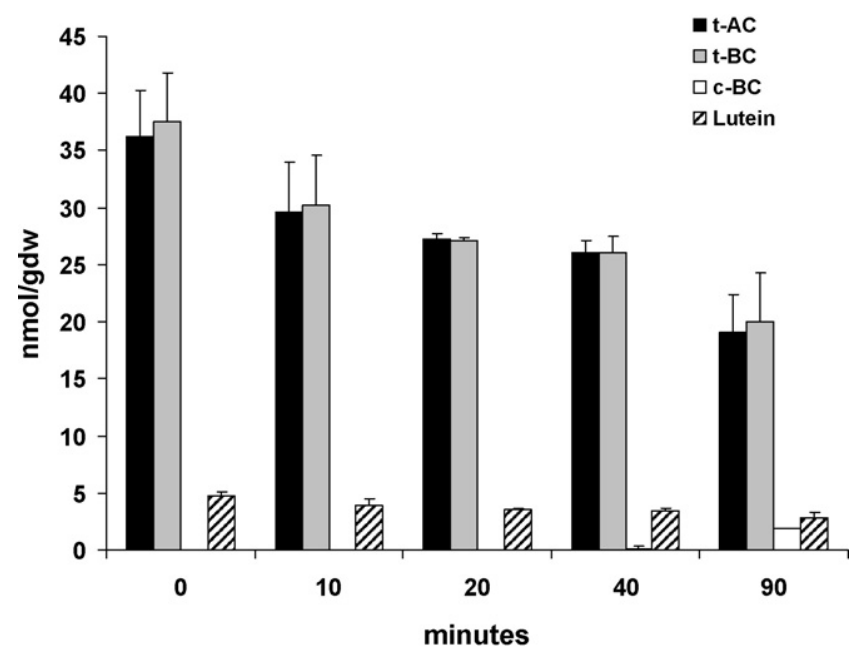

Fig. 1. Impact of a single heating step at $85^{\circ} \mathrm{C}$ during extraction on the recovery of carotenoids from the Musa variety 'Batard'. Results represent the mean following extraction of duplicate aliquots of powdered, lyophilised Musa fruit pulp and analysis by $\mathrm{C}_{30}$ RP-HPLC.

ing had little impact on chromatographic profiles and additional peaks due to carotenoid degradation were not observed (Fig. 2).

\subsection{Aeration during sample homogenisation}

Reciprocal shakers are widely used in the routine extraction of nucleotides, proteins, etc., from plant tissues. Since the strong aeration that takes place could also lead to oxidative degradation we investigated the impact of this homogenisation step on pVACs recoveries and profiles in Musa fruit. For both cultivars there was no significant difference in recoveries with a total variation across analyses for all conditions of $133.6 \pm 3.5$ and $58.0 \pm 4.6 \mathrm{nmol} / \mathrm{g} \mathrm{dw}$ for Henderneyargh and Oonoonoo kengoa, respectively. The proportions of individual carotenoids and RP-HPLC profiles were also unaffected. Therefore as standard a single homogenisation step only was included to ensure uniform homogenisation of samples.

\subsection{Stability of carotenoid extracts}

When analysing large numbers of samples, it is important to determine the stability of extracts to avoid possible losses prior to analysis. Analysis of aliquots of $0.004 \mathrm{mg} / \mathrm{mL}$ solutions of $\mathrm{t}-\mathrm{BC}$ and apocarotenal standards stored either at room temperature in the light, at room temperature in the dark or at $8^{\circ} \mathrm{C}$ in the dark clearly demonstrated that solutions stored at room temperature in

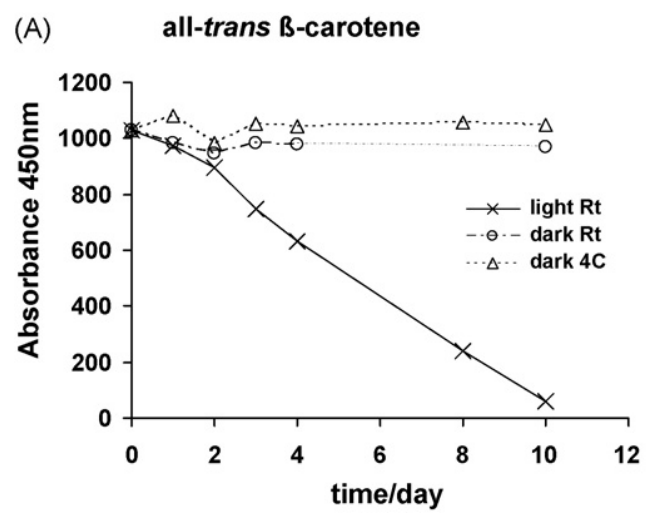

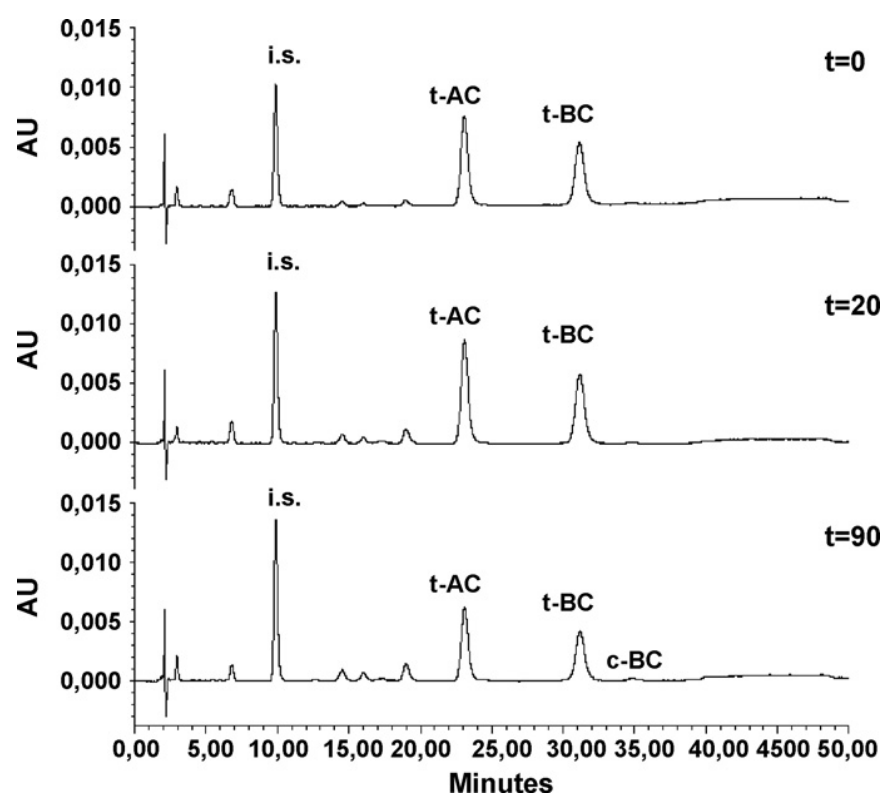

Fig. 2. Impact of a single heating step at $85^{\circ} \mathrm{C}$ for various lengths of time during extraction on the $\mathrm{C}_{30}$ RP-HPLC chromatographic profiles of carotenoid extracts from aliquots of fruit of the plantain variety 'Batard'. Eluates monitored at $450 \mathrm{~nm}$ as according to Section 2.

the light are the least stable. After 10 days in the light at room temperature, apocarotenal and t-BC solutions had lost $50 \%$ and $95 \%$ of their initial concentrations, respectively. By comparison, solutions kept in the dark retained around 90\% of the initial concentrations after 10 days, with lower temperatures improving stabilities further (Fig. 3). Again, the observed losses were not mirrored by the appearance of comparable levels of carotenoid isomers or degradation products.

We also examined the stability of $1 \mathrm{mg} / \mathrm{mL}$ stock solutions of $\mathrm{t}-\mathrm{BC}$ and apocarotenal stored at $-20^{\circ} \mathrm{C}$ in the dark. Surprisingly, the gradients of standard curves prepared from the same stock decreased significantly with time, such that after 5 days storage, gradients were only $88.4 \%$ and $89.4 \%$, respectively, of the initial values.

\subsection{Chromatographic separation methods}

RP-HPLC profiles of Musa fruit pulp carotenoids consist primarily of t-AC, t-BC, c-AC and c-BC with only small amounts of lutein $[7,9]$. Consequently, adequate separations can be obtained using both $C_{18}$ and $C_{30}$ columns. However, the greater hydrophobicity of

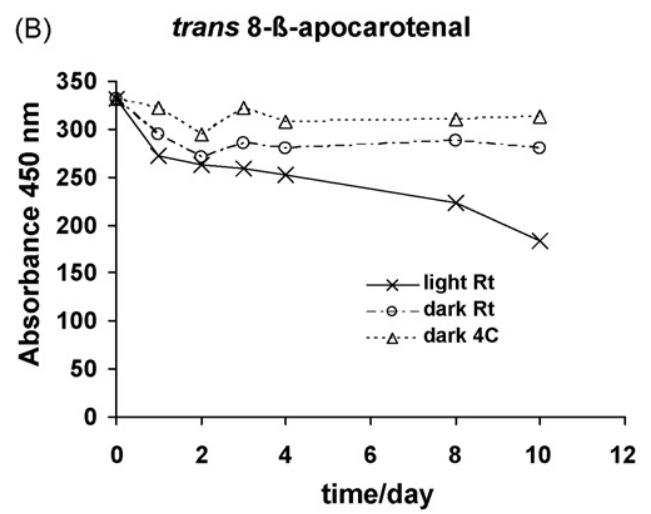

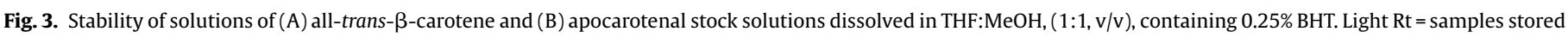
in clear glass at room temperature, dark Rt = samples stored in amber vials in the dark at room temperature, dark $4{ }^{\circ} \mathrm{C}=$ samples stored in amber vials in the dark at $4{ }^{\circ} \mathrm{C}$. 
$C_{30}$ columns allows twice as much extract to be loaded, improving detection sensitivities as well as the resolution of minor carotenoid species. To minimise analysis times on the $C_{30}$ column though it is recommended to use a gradient separation and for Musa extracts excellent resolution is obtained with a 24 min linear gradient as described in Section 2.4. Minimum detection limits are in the region of 0.5 pmol with linearity of detector response observed at least over the range 2-9000 nmol injected.

\section{Conclusions}

The chemical structures of carotenoids and pVACs makes them inherently unstable and sensitive to isomerisation and oxidation during extraction and storage. Results here indicate that many of these breakdown products are not detected by RP-HPLC so that it cannot be determined whether losses have already occurred or not during sample preparation. Consequently each step of the extraction protocol has to be carefully optimised. Here we show that neither the inclusion of PVPP to complex phenolics compounds, nor the use of a reciprocal shaker to homogenise samples during extraction had any influence on carotenoid and pVACs recoveries. By contrast the use of heat to improve extraction efficiencies resulted in losses of over $50 \%$ of all carotenoid compounds after $90 \mathrm{~min}$. Stability studies further show that exposure of extracts to light should be avoided at all costs, with $\mathrm{t}-\mathrm{BC}$ being more sensitive than the internal standard apocarotenal even in the presence of BHT. However, while extracts are reasonably stable for short periods when stored in the dark at $4{ }^{\circ} \mathrm{C}$, even at $-20^{\circ} \mathrm{C}$ in the dark, significant breakdown of concentrated stock solutions of standards occurs at a rate of about $2 \% /$ day occurs. Standard curves therefore should always be prepared fresh daily.

\section{Acknowledgements}

The authors thank Otto van Poeselaere and Eva A. Davey for help in the preparation of the manuscript and Peter Salaets for technical help. The authors acknowledge financial support from CGIAR-Bioversity and the Harvest Plus Global Challenge program on
Nutritional Enhancement and Biodiversity, as well as Inge van denBerg from Bioversity International, Montpellier, France and Gerard Bertin Ngoh-Newilah of CARBAP, Cameroon.

\section{References}

[1] P.D. Fraser, P.M. Bramley, Prog. Lipid Res. 43 (2004) 228

[2] K.J. Yeum, R.M. Russell, Annu. Rev. Nutr. 22 (2002) 483.

[3] R.M. Welch, R.D. Graham, Plant Soil 245 (2002) 205

[4] M.W. Davey, H. Garming, B. Ekesa, N. Roux, Van den Bergh I, Exploiting banana biodiversity to reduce vitamin A deficiency-related illness: a fast and cost-effective strategy, in: Proceedings, Tropical Fruits in Human Health and Nutrition Conference 2008, Brisbane, Australia, Nov 8-11, 2008.

[5] S.A. Tanumihardjo, H. Bouis, C. Hotz, J.V. Meenakshi, B. McClafferty, Comprehens. Rev. Food Sci. Food Saf. 7 (2008) 329.

[6] M.W. Davey, I. Van den Bergh, R. Markham, R. Swennen, J. Keulemans, Food Chem. 115 (2009) 806.

[7] M.W. Davey, E. Stals, G. Ngoh-Newilah, K. Tomekpe, C. Lusty, R. Markham, R. Swennen, J. Keulemans, J. Agric. Food Chem. 55 (2007) 2633.

[8] M.E. O’Neill, Y. Carroll, B. Corridan, B. Olmedilla, F. Granado, I. Blanco, H. Van den Berg, I. Hininger, A.M. Rousell, M. Chopra, S. Southon, D.I. Thurnham, Br. J. Nutr. 85 (2001) 499.

[9] M.W. Davey, J. Keulemans, R. Swennen, J. Chromatogr. A 1136 (2006) 176.

[10] A. Schieber, R. Carle, Trends Food Sci. Technol. 16 (2005) 416.

[11] O. Sommerburg, C.D. Langhans, J. Arnhold, M. Leichsenring, C. Salerno, C. Crifo, G.F. Hoffmann, K.M. Debatin, W.G. Siems, Free Radic. Biol. Med. 35 (2003) 1480

[12] M. Carail, C. Caris-Veyrat, Pure Appl. Chem. 78 (2006) 1493.

[13] E.B. Rodriguez, D.B. Rodriguez-Amaya, Food Chem. 101 (2007) 563.

[14] B.K. Dadzie, J.E. Orchard, INIBAP Technical Guidelines, International Plant Genetic Resources Institute, 1997.

[15] C.H. Azevedo-Meleiro, D.B. Rodriguez-Amaya, J. Food Compos. Anal. 17 (2004) 385.

[16] J.A. Howe, S.A. Tanumihardjo, J. Nutr. 136 (2006) 2562.

[17] J. Schierle, B. Pietsch, A. Ceresa, C. Fizet, E.H. Waysek, J. AOAC Int. 87 (2004) 1070.

[18] M. Kimura, C.N. Kobori, D.B. Rodriguez-Amaya, P. Nestel, Food Chem. 100 (2007) 1734.

[19] M. Nguyen, D. Francis, S. Schwartz, J. Sci. Food Agric. 81 (2001) 910.

[20] A.L. Vasquez-Caicedo, S. Schilling, R. Carle, S. Neidhart, Food Chem. 102 (2007) 1172.

[21] D. Qiu, Z.R. Chen, H.R. Li, Food Chem. 112 (2009) 344

[22] A. Rodríguez-Bernaldo de Quirós, H.S. Costa, J. Food Comp. Anal. 19 (2006) 97.

[23] D.B. Rodriguez-Amaya, M. Kimura, H.T. Godoy, J. Amaya-Farfan, J. Food Compos. Anal. 21 (2008) 445.

[24] E. Levizou, Y. Petropoulou, Y. Manetas, Photosynthetica 42 (2004) 295

[25] L. Englberger, J. Schierle, W. Aalbersberg, P. Hofmann, J. Humphries, A. Huang, A. Lorens, A. Levendusky, J. Daniells, G.C. Marks, M.H. Fitzgerald, Int. J. Food Sci. Nutr. 57 (2006) 399. 